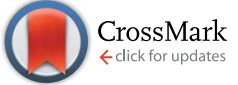

Cite this: Chem. Sci., 2016, 7, 5118

Received 19th March 2016

Accepted 10th April 2016

DOI: $10.1039 / \mathrm{c} 6 \mathrm{sc01251e}$

www.rsc.org/chemicalscience

\title{
Multilayered semiconducting polymer nanoparticles with enhanced NIR fluorescence for molecular imaging in cells, zebrafish and mice $\uparrow$
}

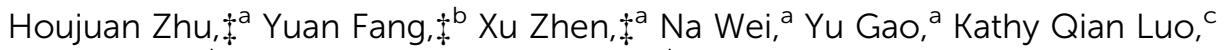 \\ Chenjie Xu, ${ }^{\text {ad }}$ Hongwei Duan, ${ }^{a}$ Dan Ding, ${ }^{* b}$ Peng Chen*a and Kanyi Pu*a
}

\begin{abstract}
Although organic semiconducting polymer nanoparticles (SPNs) have emerged as an important category of optical imaging agents, their application in molecular imaging is still in its infancy and faces many challenges. We herein report a straightforward one-pot synthetic approach to construct multilayered near-infrared (NIR) fluorescent SPNs with enhanced fluorescence and optimized biodistribution for in vivo molecular imaging. In addition to the SP core, the multilayered SPNs have a middle silica protection layer and an outer poly(ethylene glycol) (PEG) corona, which play crucial roles in enhancing the NIR fluorescence by up to $\sim 100$ fold and reducing nonspecific interactions, respectively. Their proof-ofconcept imaging applications are demonstrated in cells, zebrafish and living mice. The multilayered nanoarchitecture not only permits in vivo lymph node tracking with an ultrahigh signal-to-noise ratio ( $\sim 85)$, but also allows for more sensitive in vivo imaging of tumors with a fluorescence intensity ratio of tumor to liver that is $\sim 8$-fold higher compared to that of the counterpart silica SPN. Thus, this study provides a simple yet effective nanoengineering approach to facilitate the application of SPNs in molecular imaging.
\end{abstract}

\section{Introduction}

Molecular imaging plays a crucial role in the understanding of biology, the implementation of early diagnosis and treatment of life-threatening diseases. ${ }^{1-3}$ Among numerous imaging agents, nanoparticles have recently been investigated and proven to be effective for biomedical imaging because of their multimodal signalling capacity, improved targeting capability via multivalent binding, enhanced permeation and retention for tumor targeting, large payload delivery and tunable biodistribution profiles. ${ }^{4-9}$ Along with the numerous opportunities in medicine provided by the convergence of molecular imaging and nanotechnology, new nanoagents with improved properties in terms of biocompatibility and imaging sensitivity are desired so as to

${ }^{a}$ School of Chemical and Biomedical Engineering, Nanyang Technological University, Singapore, 637457.E-mail: chenpeng@ntu.edu.sg; kypu@ntu.edu.sg

${ }^{b}$ State Key Laboratory of Medicinal Chemical Biology, Key Laboratory of Bioactive Materials, Ministry of Education, College of Life Sciences, Nankai University, China. E-mail: dingd@nankai.edu.cn

${ }^{c}$ Faculty of Health Sciences, University of Macau, Avenida da Universidade, Taipa, Macau, China

${ }^{d}$ NTU-Northwestern Institute for Nanomedicine, Nanyang Technological University, 50 Nanyang Avenue, Singapore

$\dagger$ Electronic supplementary information (ESI) available. See DOI: 10.1039/c6sc01251e

\$ These authors contributed equally to this work. facilitate the translation of nanoparticle-related imaging technologies into clinical applications.

Semiconducting polymer nanoparticles (SPNs) constitute a new class of optical nanomaterial for molecular imaging. ${ }^{\mathbf{1 0 - 1 4}}$ Distinct from inorganic nanoparticles such as quantum dots and gold nanoclusters, SPNs are generally made from benign organic ingredients including hydrophobic semiconducting polymers (SPs) and amphiphilic polymer matrices through nanoprecipitation. ${ }^{15-19}$ Thereby, SPNs intrinsically circumvent the issue of heavy metal ion-induced toxicity to living organisms and possess good biocompatibility. ${ }^{\mathbf{2 0 , 2 1}}$ Utilization of SPNs as fluorescent nanoagents has led to a variety of applications including in vitro cell imaging, ${ }^{15,22-27}$ in vivo cell tracking, ${ }^{28}$ targeted tumor imaging, ${ }^{\mathbf{1 4 2 9 - 3 1}}$ ultrafast hemodynamic imaging, ${ }^{32}$ and drug toxicity evaluation. ${ }^{19}$ In addition, we recently revealed that SPNs can efficiently convert photon energy into heat, permitting sensitive photoacoustic imaging of reactive oxygen species (ROS), ${ }^{18,19} \mathrm{pH}$ variation ${ }^{33}$ and tumors in living animals. ${ }^{34}$ We also found that SPNs are generally resistant to ROS and much more photostable than gold nanorods, allowing for reliable imaging and sensing in living animals where ROS are ubiquitous with concentrations up to the micromolar level. Despite this progress, application of SPNs in molecular imaging is still in its infancy and faces many challenges.

One of the key challenges in advancing SPNs to in vivo imaging lies in the development of new chemistry and 
engineering methodologies that permit improved fluorescence quantum yields in the near-infrared (NIR) region without compromising other desired biophysical features. ${ }^{35}$ Doping NIR dyes into SPNs to create fluorescence resonance energy transfer (FRET) improves the NIR quantum yield, ${ }^{36,37}$ but their absorption maxima are still located in the ultraviolet and visible regions. This is not ideal for imaging in living animals because the short-wavelength excitation light has shallow tissue penetration and causes strong tissue autofluorescence. Diluting the concentration of SPs by co-precipitation with other amphiphilic copolymers can mitigate the self-aggregation of SPs and in turn increase their quantum yield to some extent; however, it lowers the absorption coefficient on a per-particle basis. ${ }^{38}$ Thereby, new designs that can improve the NIR-fluorescence of SPNs while maintaining other advantages for in vivo imaging remain elusive.

We herein report a simple yet effective synthetic approach to construct multilayered SPNs (SPNs-M) with enhanced NIR fluorescence and improved biodistribution for molecular imaging. SPNs-M consist of an inner NIR fluorescent SP core, a middle silica protection layer and an outer poly(ethylene glycol) (PEG) corona. In contrast to traditional dye-doped fluorescent silica nanoparticles with a relatively hydrophobic surface and high reticuloendothelial system (RES) uptake, ${ }^{39-44}$ SPNs-M possess an extra dense PEG cloaking corona that is formed in situ by utilization of a silane-PEG macromolecule as a surface end-capping agent during the silica cross-linking process. This straightforward synthetic approach gives the SPNs-M a unique multilayered nano-architecture, wherein the silica protection layer and the outer PEG corona play their respective roles in enhancing the NIR fluorescence and improving the in vivo biodistribution. Such a multilayer design ultimately permits sensitive imaging of the lymph nodes and tumors in living mice. We will describe a one-pot in situ synthesis of SPNs-M with a PEG shell, and then study the effect of each layer on the physical and biochemical features of the SPNs-M. Finally, we will show proof-of-concept imaging applications of these SPNs-M in cells, zebrafish and living mice.

\section{Experimental section}

\section{Materials}

All chemicals were purchased from Sigma-Aldrich unless otherwise stated. Poly[5-octyl-1-(5-(4,4,9,9-tetrakis(4-hexylphenyl)-4,9dihydro-s-indaceno[1,2- $\left.b: 5,6-b^{\prime}\right]$ dithiophen-2-yl)thiophen-2-yl)3-(thiophen-2-yl)-4H-thieno[3,4-c]pyrrole-4, 6(5H)-dione] (PDTPDTT), poly[2,6-(4,4'-bis(2-ethylhexyl) dithieno[3,2-b:2 $\left.2^{\prime}, 3^{\prime}-d\right]$ silole)alt-4,7(2,1,3-benzothiadiazole)] (PSBTBT), poly\{3-(5-(9-hexyl-9octyl-9H-fluoren-2-yl)thiophen-2-yl)-2,5-bis(2-hexyldecyl)-6-(thiophen-2-yl)pyrrolo[3,4-c]pyrrole-1,4(2H,5H)-dione $\}$ (DPPF), and poly[2,6-(4,4-bis-(2-ethylhexyl)- $4 H$-cyclopenta[2,1- $\left.b ; 3,4-b^{\prime}\right]$ dithiophene)-alt-4,7(2,1,3-benzothiadiazole)] (PCPDTBT) were purchased from Luminescence Technology Corp. Milli-Q water was supplied by Milli-Q Plus System (MilliporeCorporation, Breford, U.S.A.).

\section{Characterization}

TEM images were obtained on a JEM 1400 transmission electron microscope with an accelerating voltage from 40 to $120 \mathrm{kV}$. Dynamic light scattering (DLS) was performed using a Malvern Nano-ZS Particle Size. UV-Vis spectra were recorded on a Shimadzu UV-2450 spectrophotometer. Fluorescence measurements were carried out on a Fluorolog 3-TCSPC spectrofluorometer (Horiba JobinYvon). Fluorescence images of SPN solutions were acquired with an IVIS spectrum imaging system. Fluorescence confocal images were obtained using confocal laser scanning microscopy (Leica TSC SP8, Germany). Quantum yields of the SPNs were measured using Indocyanine Green (ICG) $\left(0.1 \times 10^{-6} \mathrm{M}\right)$ as the standard with a known quantum yield of $1 \%$ in $\mathrm{H}_{2} \mathrm{O}$.

\section{Synthesis of SPNs-M}

First, F127 (50 mg) and SPs (0.25 mg) were dissolved in THF $(1.5 \mathrm{~mL})$ in a $10 \mathrm{~mL}$ glass vial and stirred for $3 \mathrm{~h}$ at room temperature to obtain a homogeneous solution. After evaporating the THF solvent with a gentle nitrogen flow, a solid film was obtained. Then, hydrochloride solution (0.85 M, 1.6 mL) was added into the solid F127/SP mixture using bath sonication to form a homogeneous suspension. After adding TEOS $(90 \mu \mathrm{L})$, the mixture was stirred for $2 \mathrm{~h}$ at room temperature before the addition of silane-PEG2000 (50 mg). Stirring was maintained for another $24 \mathrm{~h}$ at room temperature. Finally, in order to remove hydrochloride and ethanol (resulting from the hydrolysis of TEOS and silane-PEG2000), the reaction solution was centrifuged four times at $3500 \mathrm{rpm}$ for $30 \mathrm{~min}$ using an ultrafiltration tube with a cutoff molecular weight of $50 \mathrm{~K}$. The SPN solution was filtered with a Teflon filter $(0.22 \mu \mathrm{m})$ to remove any possible large particles. DLS was conducted before filtration. These data indicate that the percentage of large nanoparticles is $0.8 \%$, proving the high reaction yield of the one-pot reaction.

\section{Synthesis of SPNs-Si}

SPNs-Si were synthesized based on a slightly modified method. Briefly, the first three steps were same as that for the SPNs-M. Subsequently, dimethoxydimethlsilane (DMDMS) $(30 \mu \mathrm{L})$ was added to the mixture instead of silane-PEG. Stirring was maintained for another $24 \mathrm{~h}$ at room temperature. Finally, in order to remove hydrochloride and ethanol (resulting from the hydrolysis of TEOS and DMDMS), the resulting solution was centrifuged a few times at $3500 \mathrm{rpm}$ for $30 \mathrm{~min}$ using an ultrafiltration tube with a cutoff molecular weight of $50 \mathrm{~K}$. The SPN solution was filtered with a Teflon filter $(0.22 \mu \mathrm{m})$ to remove any possible large particles before use.

\section{Synthesis of SPNs-F}

SPNs-F were prepared via a matrix-encapsulation method. A THF solution ( $1 \mathrm{~mL}$ ) containing F127 $(20 \mathrm{mg})$ and SPs $(0.1 \mathrm{mg})$ was quickly injected into MilliQwater $(10 \mathrm{~mL})$ and sonicated, followed by sonication of the mixture for $180 \mathrm{~s}$ at $110 \mathrm{~W}$ output using an ultrasonication bath (M2800-E, Bransonic). The mixture was then stirred at room temperature with a gentle 
nitrogen flow to evaporate the THF. The formed SPNs-F suspension were further filtered with a $0.22 \mu \mathrm{m}$ syringe filter to remove the large nanoparticles.

\section{Synthesis of SPNs-B}

SPNs-B were prepared with a simple method. Briefly, a THF solution $(1 \mathrm{~mL})$ containing SPs $(20 \mathrm{mg})$ was injected into water $(10 \mathrm{~mL})$ and sonicated. The sonication lasted for $180 \mathrm{~s}$ at $110 \mathrm{~W}$ output using an ultrasonication bath (M2800-E, Bransonic). The mixture was then stirred at room temperature with a gentle nitrogen flow to evaporate the THF. The formed SPNs-B suspension was further filtered with a $0.22 \mu \mathrm{m}$ syringe filter to remove the large nanoparticles.

\section{Cytotoxicity of SPNs-M and SPNs-Si}

HeLa cells were cultured in Dulbecco's Modified Eagle Medium (DMEM) containing $10 \%$ fetal bovine serum in a humidified environment containing $5 \% \mathrm{CO}_{2}$ and $95 \%$ air at $37{ }^{\circ} \mathrm{C}$. The metabolic activity of the HeLa cells was evaluated using methylthiazolyldiphenyl-tetrazolium (MTT) assays. HeLa cells were seeded in 96-well plates (Costar, IL, U.S.A.) at an intensity of $3 \times$ $10^{4}$ cells per $\mathrm{mL}$. After $24 \mathrm{~h}$ incubation, the medium was replaced with fresh medium containing SPNs-M or SPNs-Si suspensions at different concentrations, and the cells were then incubated for $24 \mathrm{~h}$. After a designated time interval, the medium in each well was replaced with freshly prepared MTT $(100 \mu \mathrm{L}$, $0.1 \mathrm{mg} \mathrm{mL} \mathrm{m}^{-1}$ ) solution in culture medium. The cells were incubated for $3 \mathrm{~h}$ in an incubator, followed by removal of the culture medium with MTT, and then DMSO $(100 \mu \mathrm{L})$ was added. Then, the resulting mixture was gently shaken for $10 \mathrm{~min}$ at room temperature to dissolve all the precipitate formed. The absorbance of the mixture was measured at $490 \mathrm{~nm}$ using a themicroplate reader. Cell viability was estimated using the ratio of the absorbance of the cells incubated with SPNs-M or SPNs-Si suspensions to that of the cells incubated with culture medium only.

\section{Cell imaging}

HeLa cells (human epithelial carcinoma cell line; American Type Culture Collection) were cultured in a Lab-Tek chambered cover-glass (ThermoFisher) with the growth medium (DMEM supplemented with $10 \%$ fetal bovine serum and $1 \%$ penicillinstreptomycin; ThermoFisher) at $37{ }^{\circ} \mathrm{C}$ in a humidified atmosphere containing $5 \% \mathrm{CO}_{2}$ and $95 \%$ air. The cells were, respectively, incubated for $24 \mathrm{~h}$ with SPN2-M and SPN2-Si (10 $\mu \mathrm{g}$ $\left.\mathrm{mL}^{-1}\right)$, before staining the lysosomes with LysoTracker Green (50 nM, $30 \mathrm{~min}$, ex/em-488/520 nm; ThermoFisher) and the nuclei with Hoechst 33342 (NucBlue Live ReadyProbes Reagent, ex/em-405/460 nm; ThermoFisher). Confocal images of the live cells were taken with a LSM710 confocal laser-scanning microscope (Carl Zeiss, Germany) using an excitation wavelength of $405 \mathrm{~nm}$ for Hoechst, $488 \mathrm{~nm}$ for LysoTracker Green, and $580 \mathrm{~nm}$ for the sample.

\section{Zebrafish imaging}

Imaging experiments of SPN2-M and SPN2-Si in the tissues of zebrafish larvae after microinjection were conducted in a simple way. In brief, after anesthetization of the zebrafish larvae $48 \mathrm{~h}$ post fertilization in fish medium with $0.01 \%$ tricaine, SPN2-M or SPN2-Si $\left(20 \mu \mathrm{g} \mathrm{mL}{ }^{-1}, 3 \mathrm{~nL}\right)$ was microinjected into the perivitelline space between the periderm and the yolk using microinjection equipment. $1 \mathrm{~h}$ after microinjection, bright field and fluorescence images of the zebrafish were obtained using a confocal laser microscope.

\section{In vivo subcutaneous imaging and lymph-node imaging}

All animal studies were performed in compliance with the guidelines set by Tianjin Committee of Use and Care of Laboratory Animals or the Institutional Animal Care and Use Committee (IACUC), SingHealth. Male nude mice were used for in vivo lymph-node imaging. For lymph node imaging, SPN4-M ( $30 \mu \mathrm{L}, 1 \mathrm{mg} \mathrm{mL}{ }^{-1}$ ) was administered to the forepaws of living mice anesthetized using $2 \%$ isoflurane in oxygen via intradermal injection. $4 \mathrm{~h}$ after injection, in vivo fluorescence imaging of the mice was carried out (excitation: $645 \pm 15 \mathrm{~nm}$; emission: $820 \pm 10 \mathrm{~nm}$ ).

\section{In vivo tumor imaging}

Tumor cells were harvested by incubation with $0.05 \%$ trypsinEDTA when they reached near confluence. Cells were pelleted using centrifugation and resuspended in DMEM $(10 \%$ fetal bovine serum, $1 \%$ pen per strep, $100 \mathrm{U} \mathrm{mL}^{-1}$ penicillin and 100 $\mu \mathrm{g} \mathrm{mL}{ }^{-1}$ streptomycin). $4 \mathrm{~T} 1$ cells $\left(2 \times 10^{6}\right.$ cells per site $)$ were injected subcutaneously into the right shoulder of six-week-old $\mathrm{BALB} / \mathrm{c}$ nude mice. Tumors were grown until a single aspect was $\sim 7 \mathrm{~mm}$ (approximately two weeks) before use for the imaging study. After the nude mice were anesthetized using $2 \%$ isoflurane in oxygen, SPN4-M $\left(150 \mu \mathrm{L}, 1 \mathrm{mg} \mathrm{mL}{ }^{-1}\right)(n=3)$ or SPN4Si $\left(150 \mu \mathrm{L}, 1 \mathrm{mg} \mathrm{mL}^{-1}\right)(n=3)$ was systematically injected through the tail vein using a microsyringe. Fluorescence wholeanimal imaging was performed using a CRi Maestro whole animal imaging system (Caliper Life Sciences, MA, USA). Fluorescence images of the mice were acquired at designated time points after nanoparticle administration. Image quantitation was performed using Nuance software. Mice were killed by cervical dislocation under deep isoflurane anesthesia 24 h postinjection of the nanoparticles. The tumor, heart, liver, spleen, lung, kidney, skin, and muscle were harvested for ex vivo fluorescence imaging to estimate the tissue distribution of the nanoparticles.

\section{Results and discussion}

Four SPs (PDTPDTT, PSBTBT, DPPF and PCPDTBT, chemical structures shown in Fig. 1) with strong absorption in the NIR region were chosen here so as to facilitate in vivo imaging. To reveal the functionality of each layer, four kinds of control SPNS with different nanoarchitectures were synthesized for each SP (Fig. 1a): bare SPNs (SPNs-B), SPNs made from co-precipitation with the amphiphilic triblock copolymer (PEG- $b$-PPG- $b$-PEG, 

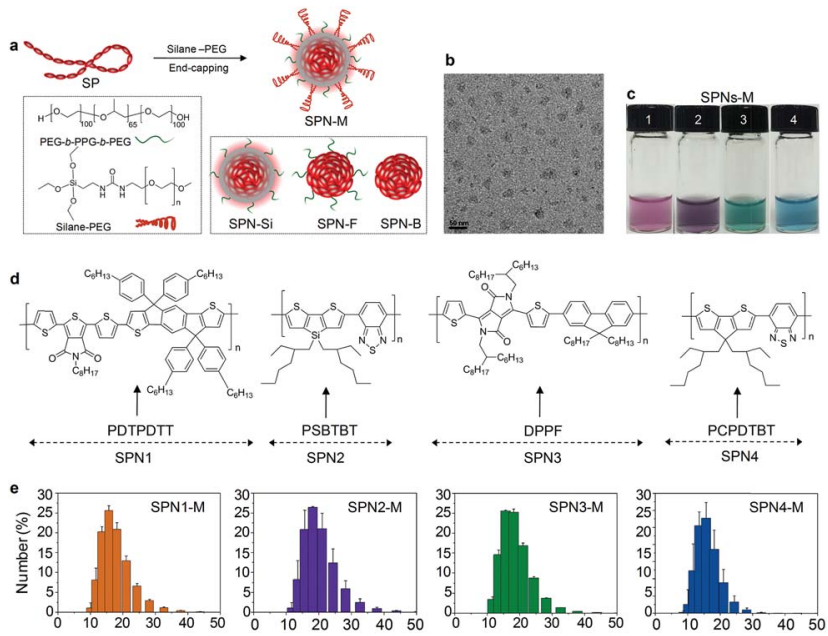

Diameter $(\mathrm{nm})$

Fig. 1 Synthesis and characterization of SPNs. (a) Schematic illustration of the preparation of SPNs-M. (b) Representative TEM image of SPNs-M: SPN4-M. (c) Photograph of the SPN-M solutions. From left to right: SPN1-M $\left(50 \mu \mathrm{g} \mathrm{mL}{ }^{-1}\right)$, SPN2-M $\left(89 \mu \mathrm{gL}^{-1}\right)$, SPN3-M $(60 \mu \mathrm{g}$ $\mathrm{mL}^{-1}$ ) and SPN4-M (45 $\left.\mu \mathrm{g} \mathrm{mL}^{-1}\right)$ in aqueous solutions. (d) Chemical structures of SP1, SP2, SP3 and SP4. (e) DLS of SPNs-M. The error bars represent the standard deviation of three separate measurements. The error bars stand for the standard deviation of three separate measurements.

F127) (SPNs-F), SPNs with a silica shell (SPNs-Si) and SPNs-M. To prepare SPNs-M, a THF solution of SPs and the amphiphilic triblock copolymer (PEG- $b$-PPG- $b$-PEG, F127) with a weight ratio of 1 : 200 was first prepared and THF was then evaporated. The amphiphilic triblock copolymer was used to stabilize the hydrophobic SP core in water as well as to serve as a template to direct the formation of the middle silica layer. After the dried film of SPs and F127 was redissolved in a hydrochloride solution with the assistance of sonication, tetraethyl orthosilicate (TEOS) was then added to the solution to allow the silicate condensation to happen, leading to the formation of the middle silica layer. Finally, silane-PEG2000 was added to the reaction solution to serve as a surface end-capping reagent to terminate the silicate condensation as well as to form the third PEG outer corona. This one-pot synthetic approach is straightforward and different from the reported preparation methods that were used to coat silica nanoparticles with PEG, which generally involved multiple post-reaction steps such as a 1-ethyl-3-(3-dimethylaminopropyl)carbodiimide (EDC) coupling reaction that may cause issues such as a broadened particle distribution and low PEG grafting density. ${ }^{45,46}$

Transmission electron microscopy (TEM) was used to examine the morphology of SPNs-M, showing uniform spherical nanoparticles with a diameter of $\sim 12 \mathrm{~nm}$ (Fig. 1c) that is close to that of SPNs-Si (Fig. S1 in the ESI $\dagger$ ). Dynamic light scattering (DLS) showed that the four SPNs-M possessed an average hydrodynamic diameter of $\sim 20 \mathrm{~nm}$ with a polydispersity of 0.21 (Fig. 1e), which was smaller than that of SPNs-Si and smaller than SPNs-F ( $\sim 30 \mathrm{~nm})$ and SPNs-B $(\sim 60)$ (Fig. S2 in the ESI $\dagger$ and Table 1). The hydrodynamic diameters were larger than the
Table 1 Summary of the absorption and emission maxima, average sizes, and quantum yields of SPNs

\begin{tabular}{llllll}
\hline Sample & & $\begin{array}{l}\lambda_{\max , \text { abs }} \\
(\mathrm{nm})\end{array}$ & $\begin{array}{l}\lambda_{\text {max,em }} \\
(\mathrm{nm})\end{array}$ & $\begin{array}{l}\text { Diameter } \\
(\mathrm{nm})\end{array}$ & $\Phi^{a}(\%)$ \\
\hline SP1 & SPN1-B & 545 & 667 & 61 & 1.64 \\
& SPN1-F & 545 & 658 & 30 & 3.02 \\
& SPN1-Si & 541 & 631 & 21 & 8.12 \\
& SPN1-M & 545 & 630 & 18 & 7.7 \\
SP2 & SPN2-B & 567 & 765 & 65 & 0.04 \\
& SPN2-F & 563 & 690 & 27 & 0.84 \\
& SPN2-Si & 540 & 615 & 21 & 4.83 \\
& SPN2-M & 540 & 640 & 19 & 4.25 \\
SP3 & SPN3-B & 609 & 700 & 67 & 0.35 \\
& SPN3-F & 612 & 690 & 29 & 1.02 \\
& SPN3-Si & 618 & 670 & 20 & 2.11 \\
SP4 & SPN3-M & 615 & 670 & 18 & 2.06 \\
& SPN4-B & 655 & 800 & 62 & 0.1 \\
& SPN4-F & 660 & 775 & 16 & 0.28 \\
& SPN4-Si & 640 & 705 & 25 & 2.46 \\
& SPN4-M & 660 & 735 & 19 & 2.28
\end{tabular}

${ }^{a}$ Fluorescence quantum yields were measured using indocyanine green (ICG) $\left(0.1 \times 10^{-6} \mathrm{M}\right)$ as the standard with a known quantum yield of $1 \%$ in $\mathrm{H}_{2} \mathrm{O}$.

diameters measured using TEM, probably because of two facts: the light scattering measurements include the contribution of the PEG chains extended in solution and the sample preparation of TEM could cause shrinkage of the nanoparticles. The size studies show that SPNs-M have a more compact nanoarchitecture relative to other counterpart nanoparticles.

To reveal the importance of the multilayer structure in enhancing the NIR quantum yield, the optical properties of SPNs-M were investigated and compared with the control nanoparticles: SPNs-B, SPNs-F and SPNs-Si. For each SP, the absorption spectra of SPNs-M, SPNs-B, SPNs-F and SPNs-Si are nearly the same (Fig. 2a and Table 1), which is ascribed to the fact that the absorption of SPNs is mainly determined by the molecular structure of the SPs inside the SP core. Comparison of the photoluminescence (PL) spectra illustrates that for all SPs

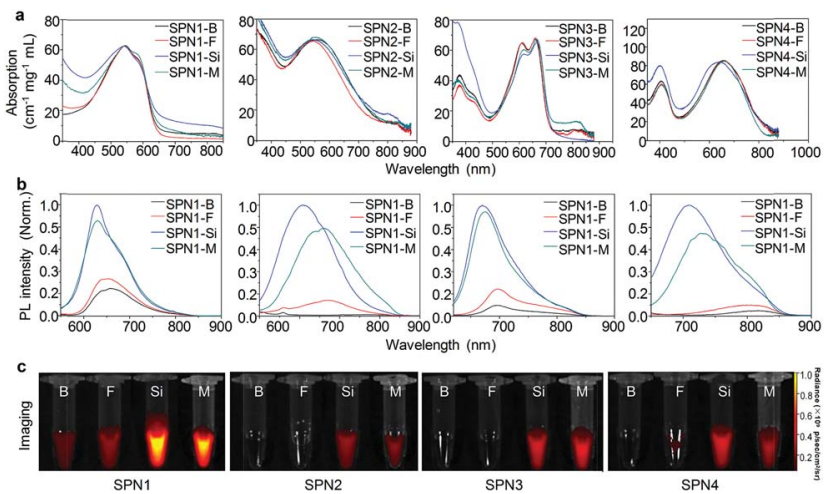

Fig. 2 Optical properties of SPNs. UV-vis absorption (a) and fluorescence (b) spectra, and IVIS fluorescence images (c) of SPNs in 1× PBS at $\mathrm{pH}=7.4$, SPN1-M: $1 \mu \mathrm{g} \mathrm{mL}^{-1}$, SPN2-M: $1.7 \mu \mathrm{g} \mathrm{mL}^{-1}$, SPN3-M: $1.2 \mu \mathrm{g}$ $\mathrm{mL}^{-1}$, and SPN4-M: $0.9 \mu \mathrm{g} \mathrm{mL}^{-1}$. 
the fluorescence intensity dramatically increases with the presence of the silica layer (Fig. 2b). The quantum yields of SPN1-M, SPN2-M, SPN3-M and SPN4-M are, respectively, 7.7\%, $4.25 \%, 2.06 \%$ and $2.28 \%$, which are, respectively, increased by $\sim 4.7,106,7.5$ and 22.8 -fold compared to their corresponding bare nanoparticle counterparts (SPNs-B). In addition, the quantum yields of SPNs-M are approximately the same as those of SPNs-Si but significantly higher than SPNs-F. This confirms that the silica layer, rather than F127 or the PEG corona, contributes to the enhanced quantum yield of SPNs-M. As all the SPs have a strong charge transfer backbone with electron donor and acceptor structural units, their fluorescence is generally sensitive to the polarity of their environment and can be quenched by increased polarity (Fig. S3 in the ESI $\dagger$ ). The silica layer in SPNs-M and SPNs-Si effectively reduces the interaction between the SP core and water molecules and provides a relatively less polar environment for the optically active SP segments compared to that in SPNs-B and SPNs-F. Such a shielding role of the silica layer consequently leads to a significant enhancement in the NIR fluorescence for SPNs-M. Note that all the SPNs are able to be excited by wavelengths above $625 \mathrm{~nm}$ and emit light above $650 \mathrm{~nm}$, which is ideal for in vivo imaging with minimized tissue autofluorescence.

The effect of the multilayer structure on the other biochemical properties of SPNs including the photostability, aqueous stability and cytocompatibility was studied (Fig. 3). The fluorescence intensity of SPNs-M shows no apparent change after continuous illumination for $80 \mathrm{~min}$ (Fig. 3a), proving their good photostability. The stability of SPNs-M in aqueous solution was investigated by monitoring the size variation using DLS. The average size remained nearly the same and no
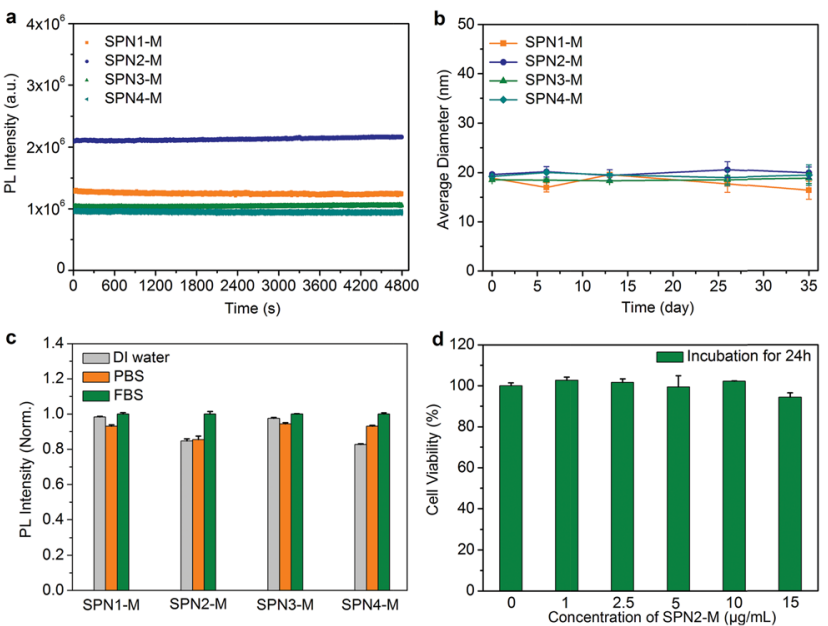

Fig. 3 In vitro stability and cytotoxicity of SPNs-M. (a) Fluorescence intensity of SPNs-M as a function of time under continuous illumination for $70 \mathrm{~min}$. (b) The hydrodynamic diameter of SPNs-M as a function of storage time. (c) Comparison of the fluorescence intensity of SPNs-M in water, PBS and FBS. (d) Cell viability of HeLa cells after incubation with SPNs-M solutions at various concentrations of SPN. (SPN1-M: $1 \mu \mathrm{g} \mathrm{mL}{ }^{-1}$, SPN2-M: $1.7 \mu \mathrm{g} \mathrm{mL}^{-1}$, SPN3-M: $1.2 \mu \mathrm{g} \mathrm{mL}^{-1}$, SPN4-M: $0.9 \mu \mathrm{g} \mathrm{mL}^{-1}$ ). The error bars represent the standard deviation from three separate measurements. precipitation was observed for SPNs-M after storage in the dark at room temperature $\left(24{ }^{\circ} \mathrm{C}\right)$ even after 35 days (Fig. 3b). Moreover, the fluorescence of SPNs-M in different media was also studied (Fig. 3c), showing that the SPNs-M had the same fluorescence intensities in water, PBS and FBS. The cytotoxicity of SPNs-M was evaluated by measuring the cell viability of HeLa cells at different SPN concentrations using 3-[4,5-dimethylthiazol-2-yl]-2,5-diphenyltetrazolium bromide (MTT) assay. The result of SPN2-M as an example is shown in Fig. 3d. The cell viability of HeLa cells is close to $90 \%$ even at a high concentration of SPN2-M (10 $\left.\mu \mathrm{g} \mathrm{mL} \mathrm{mL}^{-1}\right)$ after $24 \mathrm{~h}$ incubation. These data show that the SPNs-M have similar optical and biochemical properties relative to SPNs-Si (Fig. S4 and S5 in the ESI $\dagger$ ), proving that the multilayered nanoarchitecture has no negative influence on the photostability, aqueous stability and cytocompatibility of SPNs.

To demonstrate the capability of SPNs-M in cell imaging, the cellular uptake behavior of SPNs-M was studied and compared with its silica counterpart (SPNs-Si) using confocal laser scanning microscopy (CLSM). SPN2-M was chosen as an example because (i) it has the highest fluorescence enhancement (106-fold) after construction of the multilayer nano-architecture among these SPs, and (ii) its absorption and emission wavelengths match well with the filter parameters of commercial CLSM. After incubation with SPNs for $24 \mathrm{~h}$, the cells were washed and stained with LysoTraker Green and Hoechst to label the lysosome and nuclei in the cells with green and blue fluorescence, respectively. As shown in Fig. 4, both SPN2-M and SPN2-Si can enter the cells as witnessed by the red fluorescence from the cytoplasm. Note that there is no red autofluorescence observed in the control cells under the same CLSM parameters. The overlaid images further show that the red fluorescence overlaps with the green fluorescence from LysoTraker Green, indicating that both SPNs are mainly located in the lysosome. Imaging quantification in Fig. 4c reveals that the fluorescence intensity for SPN2-M-treated cells is 2.6-fold lower compared to that of SPN2-Si-treated cells, implying

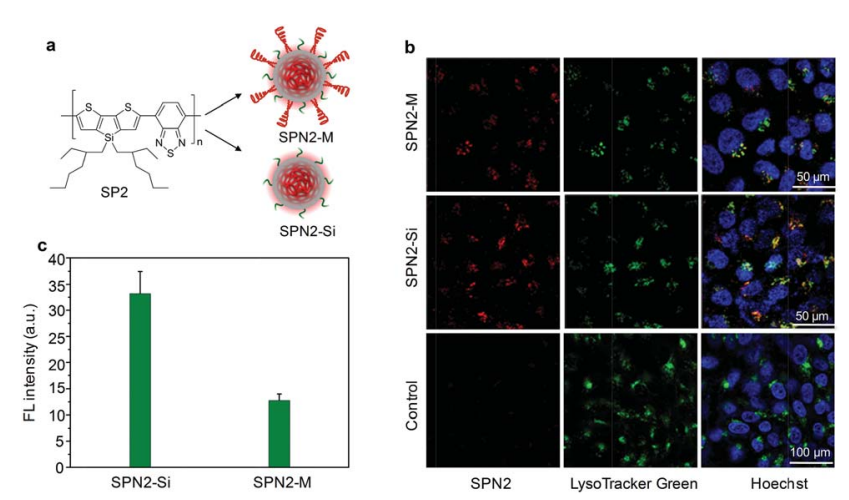

Fig. 4 Cell imaging of SPNs. (a) Schematic illustration of the preparation of SPN2-M through silica growth and silane-PEG capping. (b) Confocal laser scanning microscopy (CLSM) images of HeLa cells after $24 \mathrm{~h}$ incubation with SPN2-Si and SPN2-M (10 $\mu \mathrm{g} \mathrm{mL}^{-1}$ based on SP2). (c) Comparison of fluorescence intensities of HeLa cells stained with SPN2-Si and SPN2-M. The error bars represent the standard deviation from three separate measurements. 
a lower cellular uptake of SPN2-M relative to SPN2-Si. This is attributed to the dense PEG corona of SPN2-M that minimizes nonspecific protein adsorption and cellular uptake, which is consistent with previous studies which showed that PEGylation reduces NP uptake by cultured cells. ${ }^{47,48}$

The in vivo imaging potential of SPNs-M was evaluated with a zebrafish larvae model. Note that although SPNs with silica coating have been reported, they do not have a PEG corona layer. In addition, those silica SPNs only absorb and emit in the visible wavelength region and their in vivo applications have not been explored. ${ }^{\mathbf{3 6 , 4 9 , 5 0}}$ After microinjection of SPN2-M or SPN2-Si $\left(20 \mu \mathrm{g} \mathrm{mL}^{-1}, 3 \mathrm{~nL}\right.$ ) into zebrafish larvae for $18 \mathrm{~h}$, no abnormal morphology and pericardium edema were observed. This is consistent with the good biocompatibility of SPNs shown in the cell culture (Fig. 3d and S5 in the ESI $\dagger$ ). CLSM was conducted to image the distribution of both SPNs in the zebrafish larvae that were genetically engineered to have eGFP-expressing endothelial cells (nEGFP) in the blood vessel walls. As shown in Fig. 5a, red fluorescence can be detected for both SPNs in the yolk sac of the zebrafish larvae, while no red fluorescence is present in the control. This illustrates the similar accumulation of both SPNs in the yolk sac of the zebrafish larvae. Imaging quantification shows that the average fluorescence intensity for SPN2-M injected zebrafish is 1.14-fold higher than that for SPN2-Si injected zebrafish (Fig. 5b). This could be due to the dense PEG corona of SPN2-M that leads to the higher accumulation time in zebrafish larvae relative to that for SPN2-Si.

Lymph node tracking is clinically important in guiding the surgical resection of tumor tissues. ${ }^{51}$ As nanoparticles with diameters ranging from 20 to $50 \mathrm{~nm}$ have favourable accumulation and retention in draining lymph nodes, ${ }^{52}$ SPNs-M were then tested for lymph node tracking in living mice. SPN4-M was chosen for imaging in living mice considering its longest excitation and emission wavelengths as well as its relatively high NIR quantum yield among the four SPs (Table 1). After intradermal injection of SPN4-M into the forepaw of living mice for $1 \mathrm{~h}$, the axillary lymph node could be readily visualized with strong NIR fluorescence (Fig. 6b). Quantification shows that the signal-to-noise ratio is as high as $\sim 85$ (Fig. $6 \mathrm{~b}$ ). This is a benefit of the long-wavelength NIR excitation and emission of SPN4-M which greatly minimizes the tissue autofluorescence.

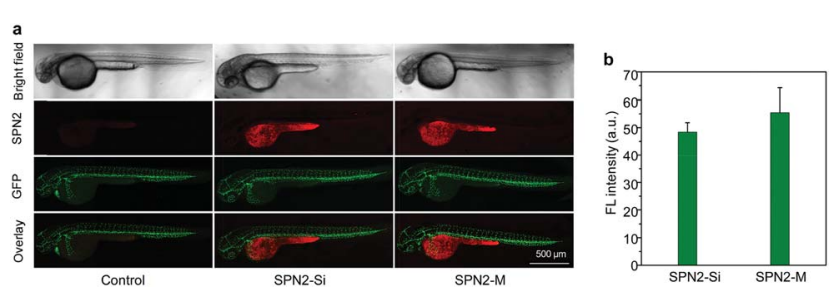

Fig. 5 Fluorescence imaging of SPNs in Tg (fli1: nEGFP) zebrafish larvae. (a) CLSM images of zebrafish larvae $48 \mathrm{~h}$ post fertilization after microinjecting SPN2-Si or SPN2-M (20 $\left.\mathrm{g} \mathrm{mL}^{-1}, 3 \mathrm{~nL}\right)$ for $18 \mathrm{~h}$. (b) Quantification of the fluorescence intensities of SPN2-Si or SPN2-M in the yolk of the zebrafish. The error bars represent the standard deviation from three separate measurements.
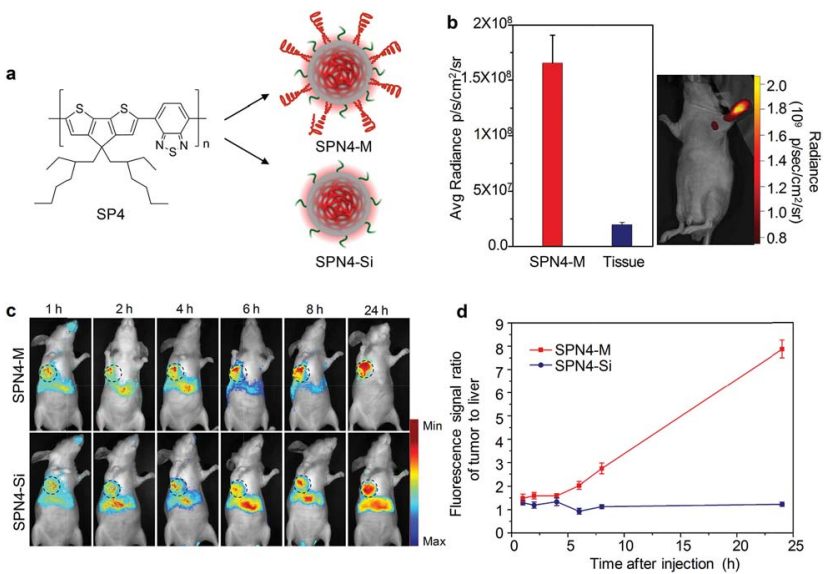

Fig. 6 In vivo fluorescence imaging. (a) Schematic illustration of the preparation of SPN4-M through silica growth and silane-PEG surface capping. (b) Left: quantification of the fluorescence intensity of lymph nodes and normal tissue in living mice $4 \mathrm{~h}$ after the intradermal injection of SPN4-M into the forepaw. Right: whole-animal fluorescence imaging of a living mouse $4 \mathrm{~h}$ after intradermal injection of SPN4-M (1 mg mL $\mathrm{mb}^{-1}, 30 \mu \mathrm{L}$ ) into the forepaw. (c) Real-time fluorescence imaging of 4T1 tumors in living mice after the systemic administration of SPN4-Si or SPN4-M (1 $\left.\mathrm{mg} \mathrm{mL}^{-1}, 150 \mu \mathrm{L}\right)$ through intravenous injection. The black dashed circles indicate the location of the tumor. (d) The fluorescence signal ratio of the tumor to the liver as a function of post-injection time for SPN4-Si or SPN4-M administrated mice. The error bars represent the standard deviation $(n=3)$.

The feasibility of using SPN4-M for tumor imaging in living mice was validated and compared with SPN4-Si. After intravenous injection of SPN4-M or SPN4-Si into 4T1-bearing nude mice, realtime whole-animal fluorescence imaging was conducted longitudinally. Note that the tumor was lightened up by both SPNS even just $1 \mathrm{~h}$ post-injection and the fluorescence intensity in the tumor area increased over time (Fig. 6c). This indicates the passive tumor targeting capability for both SPNs due to their small size that favours the enhanced permeability and retention (EPR) effect, which is consistent with our previous studies. ${ }^{33,34}$ Although the fluorescence intensity at the tumor site is almost the same for SPN4-M and SPN4-Si for the initial $8 \mathrm{~h}$ post-administration, the fluorescence intensity in the liver for SPN4-M is much lower compared to that for SPN4-Si (Fig. 6c). This implies a reduced RES uptake and improved biodistribution for SPN4-M due to the in situ formed PEG cloaking corona of SPN4-M. As such, SPN4-M can delineate the tumor in vivo with a fluorescence intensity that is 1.68 -fold brighter than SPN4-Si $24 \mathrm{~h}$ postadministration. In addition, the fluorescence intensity ratio of the tumor to the liver for SPN4-M is 8 , which is $\sim 8.0$-fold higher compared to that for SPN4-Si (1.3) (Fig. 6d). This was also confirmed using ex vivo fluorescence imaging of the major organs (Fig. S6 in the ESI $\dagger$ ). Thus, these data clearly show the advantage of a multilayered nanoarchitecture for in vivo tumor imaging.

\section{Conclusion}

We have synthesized a series of NIR-fluorescent SPNs with a multilayered nanoarchitecture to advance SPNs for in vivo 
molecular imaging. SPNs-M are synthesized via a straightforward one-pot method that permits in situ formation of a dense PEG corona on the silica layer. Such a synthetic approach results in a compact and uniform nanostructure for SPNs-M which possess a hydrodynamic size $(\sim 20 \mathrm{~nm})$ that is smaller than bare and polymer-encapsulated SPNs (SPNs-B and SPNs-F). The importance of a multilayered structure for SPNs-M is systematically studied by comparing their optical and biochemical properties with their counterpart SPNs (SPNs-B, SPNs-F and SPNs-Si). Without compromising the excellent photostability and good biocompatibility of the SP core, the middle silica layer of SPNs-M plays an essential role in enhancing the NIR fluorescence by up to 100 -fold depending on the structure of the SP, while the outer cloaking PEG corona effectively reduces the nonspecific cellular uptake and RES uptake.

The proof-of-concept application of SPNs-M as fluorescent agents has been demonstrated in living subjects ranging from cells to zebrafish to living mice. In addition to the applicability of SPNs-M in cellular and zebrafish imaging, their enhanced NIR fluorescence and improved bio-distribution not only allows for in vivo lymph node tracking with an ultrahigh signal-tonoise ratio $(\sim 85)$, but also permits more sensitive in vivo imaging of tumors with a fluorescence intensity ratio of tumor to liver that is $\sim 8.0$ times higher compared to that for the counterpart silica SPN. Thereby, this study provides a simple yet effective nanoengineering approach to advance SPNs for molecular imaging.

\section{Acknowledgements}

This work was supported by Nanyang Technological University start-up grant (NTU-SUG: M4081627.120), Academic Research Fund Tier 2 from Ministry of Education (Singapore) (MOE2014T2-1-003) and Academic Research Fund Tier 1 from Singapore Ministry of Education (M4011559.120, RG133/15). DD acknowledges the National Basic Research Program of China (2015CB856503) for support. XCJ thanks NTU-Northwestern Institute for Nanomedicine (M4081502.F40).

\section{References}

1 R. Weissleder and M. J. Pittet, Nature, 2008, 452, 580-589.

2 R. Weissleder, Science, 2006, 312, 1168-1171.

3 E. A. Osborn and F. A. Jaffer, Curr. Opin. Cardiol., 2008, 23, 620-628.

4 T. F. Massoud and S. S. Gambhir, Genes Dev., 2003, 17, 545580.

5 H. Kobayashi, M. R. Longmire, M. Ogawa and P. L. Choyke, Chem. Soc. Rev., 2011, 40, 4626-4648.

6 V. Wagner, A. Dullaart, A. K. Bock and A. Zweck, Nat. Biotechnol., 2006, 24, 1211-1217.

7 H. S. Choi, W. Liu, F. Liu, K. Nasr, P. Misra, M. G. Bawendi and J. V. Frangioni, Nat. Nanotechnol., 2010, 5, 42-47.

8 Z. Cheng, A. Al Zaki, J. Z. Hui, V. R. Muzykantov and A. Tsourkas, Science, 2012, 338, 903-910.
9 K. K. Ng, J. F. Lovell and G. Zheng, Acc. Chem. Res., 2011, 44, 1105-1113.

10 J. H. Moon, W. McDaniel, P. Maclean and L. F. Hancock, Angew. Chem., Int. Ed., 2007, 46, 8223-8225.

11 J. Pecher and S. Mecking, Chem Rev., 2010, 110, 6260-6279.

12 C. Zhu, L. Liu, Q. Yang, F. Lv and S. Wang, Chem. Rev., 2012, 112, 4687-4735.

13 K. Pu and B. Liu, Adv. Funct. Mater., 2011, 21, 3408-3423.

14 C. Wu and D. T. Chiu, Angew. Chem., Int. Ed., 2013, 52, 30863109.

15 P. Howes, M. Green, J. Levitt, K. Suhling and M. Hughes, J. Am. Chem. Soc., 2010, 132, 3989-3996.

16 J. Pecher and S. Mecking, Chem. Rev., 2010, 110, 6260-6279.

17 K. Pu, A. J. Shuhendler, J. V. Jokerst, J. Mei, S. S. Gambhir, Z. Bao and J. Rao, Nat. Nanotechnol., 2014, 9, 233-239.

18 K. Pu, A. J. Shuhendler and J. Rao, Angew. Chem., Int. Ed., 2013, 52, 10325-10329.

19 A. J. Shuhendler, K. Pu, L. Cui, J. P. Uetrecht and J. Rao, Nat. Biotechnol., 2014, 32, 373-380.

20 M. Palner, K. Pu, S. Shao, J. Jeon and J. Rao, Angew. Chem. Int. Ed., 2015, 127, 11639-11642.

21 L. Xu, L. Cheng, C. Wang, R. Peng and Z. Liu, Polym. Chem., 2014, 5, 1573-1580.

22 C. Wu, T. Schneider, M. Zeigler, J. Yu, P. G. Schiro, D. R. Burnham, J. D. McNeill and D. T. Chiu, J. Am. Chem. Soc., 2010, 132, 15410-15417.

23 C. Wu, Y. Jin, T. Schneider, D. R. Burnham, P. B. Smith and D. T. Chiu, Angew. Chem., Int. Ed., 2010, 49, 9436-9440.

24 X. Feng, Y. Tang, X. Duan, L. Liu and S. Wang, J. Mater. Chem., 2010, 20, 1312-1316.

25 P. J. Wu, S. Y. Kuo, Y. C. Huang, C. P. Chen and Y. H. Chan, Anal. Chem., 2014, 86, 4831-4839.

26 K. Sun, H. Chen, L. Wang, S. Yin, H. Wang, G. Xu, D. Chen, X. Zhang, C. Wu and W. Qin, ACS Appl. Mater. Interfaces, 2014, 6, 10802-10812.

27 X. Feng, G. Yang, L. Liu, F. Lv, Q. Yang, S. Wang and D. Zhu, Adv. Mater., 2012, 24, 637-641.

28 K. Pu, A. J. Shuhendler, M. P. Valta, L. N. Cui, M. Saar, D. M. Peehl and J. H. Rao, Adv. Healthcare Mater., 2014, 3, 1292-1298.

29 L. Xiong, A. J. Shuhendler and J. Rao, Nat. Commun., 2012, 3, 1193.

30 K. Li, D. Ding, D. Huo, K. Pu, T. Ngo Nguyen Phuong, Y. Hu, Z. Li and B. Liu, Adv. Funct. Mater., 2012, 22, 3107-3115.

31 C. Wu, S. J. Hansen, Q. Hou, J. Yu, M. Zeigler, Y. Jin, D. R. Burnham, J. D. McNeill, J. M. Olson and D. T. Chiu, Angew. Chem., Int. Ed., 2011, 50, 3430-3434.

32 G. Hong, Y. Zou, A. L. Antaris, S. Diao, D. Wu, K. Cheng, X. Zhang, C. Chen, B. Liu, Y. He, J. Z. Wu, J. Yuan, B. Zhang, Z. Tao, C. Fukunaga and H. Dai, Nat. Commun., 2014, 5, 4206.

33 Q. Miao, D. Ding and K. Pu, Adv. Mater., 2016, DOI: 10.1002/ adma.201505681.

34 Y. Lyu, Q. Miao, X. Zhen, D. Ding and K. Pu, ACS Nano, 2016, DOI: 10.1021/acsnano.6b00168.

$35 \mathrm{~K} . \mathrm{Pu}, \mathrm{N}$. Chattopadhyay and J. Rao, J. Controlled Release, 2016, DOI: 10.1016/j.jconrel.2016.01.004. 
36 J. Geng, Z. Zhu, W. Qin, L. Ma, Y. Hu, G. G. Gurzadyan, B. Z. Tang and B. Liu, Nanoscale, 2014, 6, 939-945.

37 Y. Jin, F. Ye, M. Zeigler, C. Wu and D. T. Chiu, ACS Nano, 2011, 5, 1468-1475.

38 M. B. Zeigler, W. Sun, Y. Rong and D. T. Chiu, J. Am. Chem. Soc., 2013, 135, 11453-11456.

39 M. Montalti, L. Prodi, E. Rampazzo and N. Zaccheroni, Chem. Soc. Rev., 2014, 43, 4243-4268.

40 H. Tan, N. S. Liu, B. He, S. Y. Wong, Z.-K. Chen, X. Li and J. Wang, Chem. Commun., 2009, 6240-6242.

41 H. Ow, D. R. Larson, M. Srivastava, B. A. Baird, W. W. Webb and U. Wiesner, Nano Lett., 2005, 5, 113-117.

42 Q. S. Huo, J. Liu, L. Q. Wang, Y. B. Jiang, T. N. Lambert and E. Fang, J. Am. Chem. Soc., 2006, 128, 6447-6453.

43 H. Y. Zeng, W. M. Huang and J. L. Shi, Chem. Commun., 2006, 880-881.

44 W. R. Zhao, J. L. Gu, L. X. Zhang, H. R. Chen and J. L. Shi, J. Am. Chem. Soc., 2005, 127, 8916-8917.
45 Y. S. Lin and C. L. Haynes, J. Am. Chem. Soc., 2010, 132, 48344842.

46 F. H. Chen, L. M. Zhang, Q. T. Chen, Y. Zhang and Z. J. Zhang, Chem. Commun., 2010, 46, 8633-8635.

47 J. V. Jokerst, T. Lobovkina, R. N. Zare and S. S. Gambhir, Nanomedicine, 2011, 6, 715-728.

48 J. Xie, C. Xu, N. Kohler, Y. Hou and S. Sun, Adv. Mater., 2007, 19, 3163-3166.

49 C. F. Wu, C. Szymanski and J. McNeill, Langmuir, 2006, 22, 2956-2960.

50 H. Tan, Y. Zhang, M. Wang, Z. Zhang, X. Zhang, A. M. Yong, S. Y. Wong, A. Y. Chang, Z. K. Chen, X. Li, M. Choolani and J. Wang, Biomaterials, 2012, 33, 237-246.

51 S. Kim, Y. T. Lim, E. G. Soltesz, A. M. De Grand, J. Lee, A. Nakayama, J. A. Parker, T. Mihaljevic, R. G. Laurence, D. M. Dor, L. H. Cohn, M. G. Bawendi and J. V. Frangioni, Nat. Biotechnol., 2004, 22, 93-97.

52 M. Nakajima, M. Takeda, M. Kobayashi, S. Suzuki and N. Ohuchi, Cancer Sci., 2005, 96, 353-356. 